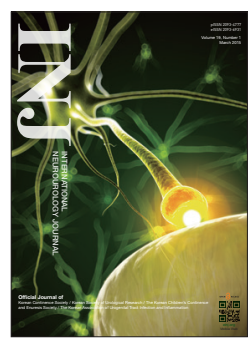

\title{
Postoperative Ureteral Leak Treated Using a Silicone-Covered Nitinol Stent
}

\author{
Hyo Jung Park ${ }^{1}$, Ji Hoon Shin ${ }^{1}$, Jong Woo Kim¹, Bum Sik Hong ${ }^{2}$ \\ ${ }^{1}$ Department of Radiology and Research Institute of Radiology, Asan Medical Center, University of Ulsan College of Medicine, Seoul, Korea \\ ${ }^{2}$ Department of Urology, Asan Medical Center, University of Ulsan College of Medicine, Seoul, Korea
}

\begin{abstract}
Ureteral fistula is a serious complication of abdomino-pelvic surgeries, often resulting in poor outcomes owing to lack of proper treatment. We report the case of a 49-year-old woman who underwent placement of a silicone-covered ureteral occlusion stent in her right ureter for the management of ureteral leakage after pelvic surgery. A ureterogram obtained 18 months following the stent placement confirmed that there was no stent migration or additional urine leakage. We propose that the silicone-covered ureteral occlusion stent is practical, fast, and safe for the management of ureteral leakage.
\end{abstract}

Keywords: Urinary Fistula; Urinary Diversion; Stents; Therapeutic Occlusion

- Conflict of Interest: No potential conflict of interest relevant to this article was reported.

Ureteral leakage, an uncommon complication of abdominopelvic surgeries, can result in some serious conditions like ureterovaginal fistula, sepsis and renal failure. Permanent transrenal ureteral diversion should be considered as a treatment option for patients with poor general state or surgically unmanageable anatomic defect of the ureter. We introduce a case of ureteral leakage which was successfully managed by the percutaneous insertion of the silicone-covered ureteral occlusion stent.

\section{CASE REPORT}

A 49-year-old woman diagnosed with stage IV ovarian cancer underwent extensive debulking surgery. Due to the presence of right hydronephrosis, a right nephrostomy tube was inserted prior to the second surgery. During surgery, additional excision was performed because of ovarian tumor invasion of the blad- der and right ureter. Her right distal ureter was partly resected; the distal margin of the remaining ureter was at the L5 level. As the remaining ureter was not long enough for anastomosis and the level of the injury site was high, ureteroureterostomy or ureterocystostomy were not possible. Therefore, ureter ligation was performed by suturing the ureter three times and clipping it once. Absence of urine outflow through the ureter was confirmed after the procedure. However, four days postsurgery, an antegrade ureterogram through the nephrostomy tube showed contrast extravasation at the site of ligation.

We believed that the ureter was unlikely to seal up spontaneously owing to the relatively large size of the wall defect seen on the ureterogram. Considering the diameter of the ureter at the transection site, the high level of the distal margin of the remaining ureter, and the patient's generally poor condition, an intervention consisting of complete ureter occlusion was planned. A special ureteral occlusion stent was ordered for the

Corresponding author: Ji Hoon Shin (iD http://orcid.org/0000-0001-6598-9049 Department of Radiology and Research Institute of Radiology, Asan Medical Center, University of Ulsan College of Medicine, 86, Asanbyeongwon-gil, Songpa-gu, Seoul 138-736, Korea

E-mail: jhshin@amc.seoul.kr / Tel: +82-2-3010-4380 / Fax: +82-2-476-0090

Submitted: January 17, 2015 / Accepted after revision: March 3, 2015 
remaining ureter.

The occlusion stent was made of nickel/titanium alloy, and

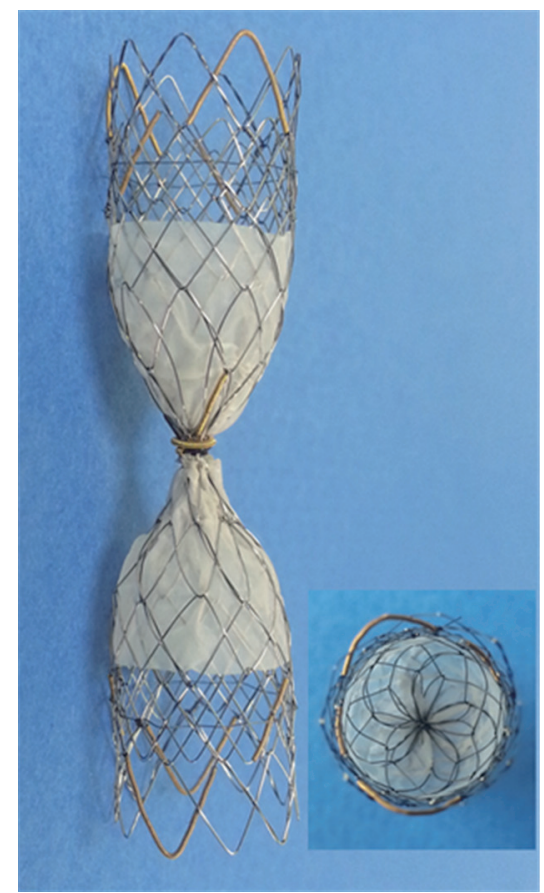

Fig. 1. A specially designed ureteral occlusion stent having candy-wrapper configuration. The middle of the stent was completely constricted in order to allow waterproofing. was super-elastic and self-expandable. The middle of the stent was constricted in a candy-wrapper configuration, leading to complete obstruction of the central portion (Fig. 1). When fully expanded, the stent was $10 \mathrm{~mm}$ in diameter and $4 \mathrm{~cm}$ in length. The stent was internally coated with a thin silicone membrane, leaving a bare portion at either end; the bare portion at the distal end was intended to permit tissue growth through the stent interstices and thus prevent stent migration. The stent was constructed according to our specifications by a local manufacturer (S\&G Biotech, Seongnam, Korea).

With the patient in a prone position, the right nephrostomy tube was replaced with a 9-Fr vascular sheath (Pinnacle TIF Tip, Terumo, Tokyo, Japan). The exact leakage site at the distal margin of the ureter was confirmed with contrast-medium injection through a 5-Fr Kumpe catheter (Cook, Bloomington, IN, USA). Using a guidewire, an 8-Fr introducer sheath was passed through the 9-Fr vascular sheath, into the ureter. The self-expandable stent was then deployed by pulling back the introducer sheath. Stent placement was technically successful and there were no immediate procedure-related complications (Fig. $2 \mathrm{~A}, \mathrm{~B})$. Injection of contrast medium through the ureter showed no contrast material passing beyond the occlusion stent, and no further ureteral leakage (Fig. 2C).

The nephrostomy tube has since been maintained. An 18-month follow-up antegrade ureterogram showed neither contrast-me-
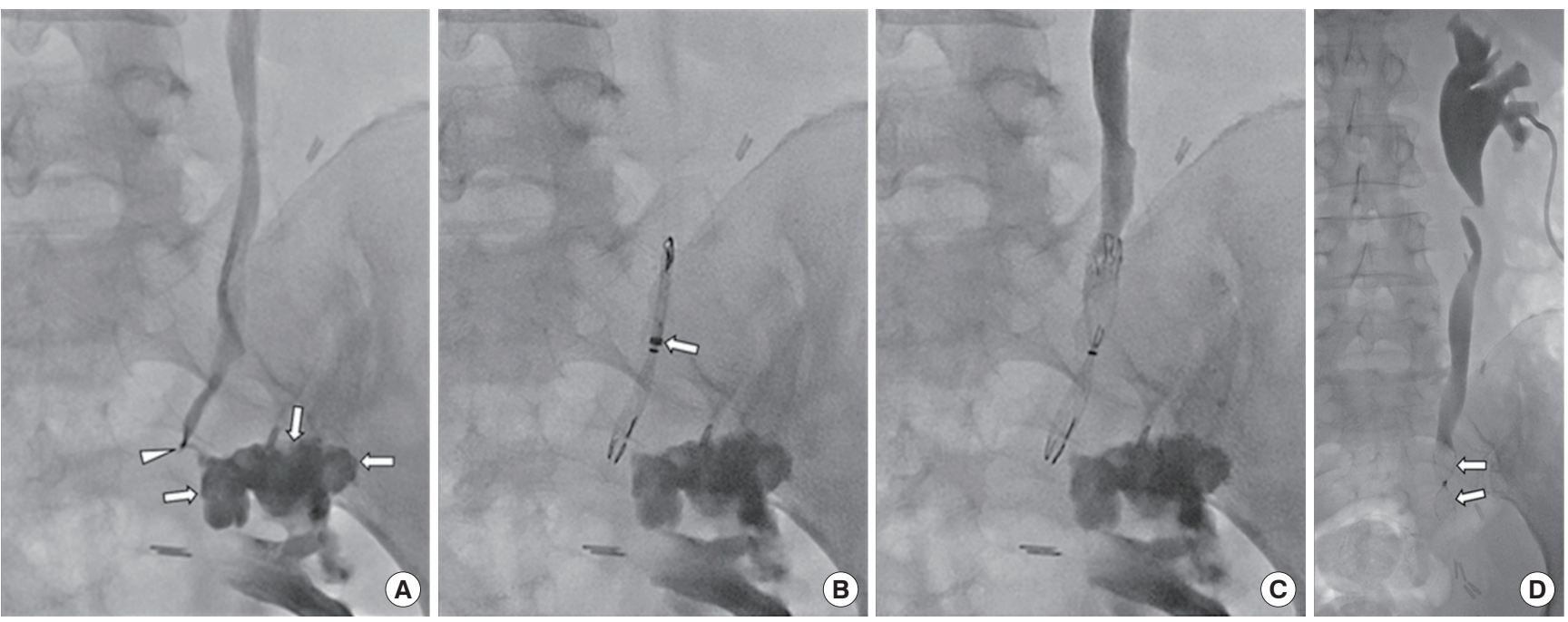

Fig. 2. Percutaneous placement of the ureteral occlusion stent. (A) Right antegrade ureterogram through the nephrostomy tube shows contrast-medium leakage (arrows) from the distal tip (arrowhead) of the right ureter. (B) The ureteral occlusion stent is deployed by pulling back the introducer sheath, which has a radiopaque distal tip (arrow). (C) Ureterogram obtained immediately following stent deployment shows complete occlusion of the ureter without further contrast-medium leakage. (D) An 18-month follow-up ureterogram shows neither contrast-medium leakage nor occlusion stent migration (arrows). 
dium leakage nor migration of the occlusion stent (Fig. 2D).

\section{DISCUSSION}

Although ureteral leakage is an uncommon complication following abdomino-pelvic surgeries or procedures, it can lead to serious conditions such as ureterovaginal fistula, intra-abdominal sepsis, renal failure, and loss of the ipsilateral kidney [1]. Numerous approaches have been attempted for the management of ureteral leakage, depending on the patient's general condition and the anatomic location of the leakage. In general, surgical management should be considered as the final option since repeated surgery could be harmful if the patient is unstable. In many cases, there are very limited reconstructive options available, considering the extent and location of the injury [2].

Transrenal ureter occlusion with permanent urinary diversion may be indicated as a palliative treatment for patients with a surgically or endourologically unmanageable anatomic defect of the ureter. Several techniques have been suggested using tissue adhesive, balloons, coils, electrocautery, stents, and siliconeoccluding devices, but all have varying degrees of success. Most of these devices and techniques were initially developed for vascular embolization. The reasons for incomplete occlusion of ureteral leakage have been continuous urinary flow at a blockage site, recanalization of an occlusion, or migration of the inserted materials [3]. In the vascular system, the coagulation cascade is triggered around a foreign device, which finally leads to the desired obstruction. However, as there are no platelets or clotting factors in normal urine, some additional anchoring, such as a fixing component or a self-expandable feature, would be necessary for the device to remain in the urinary tract without migration. Here, we review various techniques reported till date.

Isobutyl-2-cyanoacrylate has previously been used for intravascular embolization. Gunther et al. [4] reported its use in several patients, for achieving complete blockage of ureteral fistula via the transrenal approach. However, Moldwin and Smith [5] subsequently announced that the results with isobutyl-2-cyanoacrylate were inconsistent, because the material gradually softens and may allow migration by ureteral peristalsis.

Two approaches of balloon occlusion have been reportedusing detachable balloons and nondetachable balloons. In 7 patients, Gunther et al. [6] successfully used detachable balloons inflated with a silicone mixture, and reported complete ureteral occlusion without complications at 1-6 months. Additionally, Papa- nicolaou et al. [7] described the application of nondetachable balloons for temporary ureteral occlusion. Although no complications have been reported following the procedures, long-term use of balloons carries potential risks for ureteral necrosis or rupture.

The outcome of using coils and gelatin sponges in $22 \mathrm{pa}-$ tients, were described by Farrell et al. [8]. Except for four patients who died owing to progression of underlying malignancy, all the other patients remained symptom-free throughout the follow-up period ranging from 2 to 29 months. Coils may produce foreign body reactions in ureters, namely localized inflammation with the ingrowth of granulation tissues. Ureteral occlusion with coils has been reported to have relatively low complication rates compared with other methods. Reported complications include coil migration and urinary infection. Recently, the latex-covered Amplatzer vascular plug (AVP) showed promising results in the occlusion of ureteral leakage [9]; in the study, 9 of 10 ureters were completely sealed off as the expandable characteristics of AVPs and the latex that covers AVPs before implantation seemed to provide an effective watertight barrier.

Metallic stents have generally been used for retaining the patency of vessels and nonvascular luminal structures. They have also been applied to the urinary tract, and there is a high tendency for obstruction due to reactive tissue hyperplasia. We paid attention to this occlusive mechanism and designed our occlusion stent. To our knowledge, there is no report about the specific shape and function of a ureteral metallic stent designed based on the occlusive principle.

In our study, the expandable characteristics of the nitinol stent frame, as well as the silicone membrane with midline constriction, resulted in a completely watertight arrangement. In addition, the bare metallic portions at both ends might precipitate tissue ingrowth through the mesh of the stent, allowing the desirable ureteral occlusion. Moreover, the combination of the expansile feature of the nitinol and the two ends with bare metallic portion in our stent would serve as an antimigration system. This would make it superior to some other devices using only the expansile characteristic to prevent migration. In addition, the distinctive candy-wrapper configuration would prevent the separation of the silicone membrane and stent meshes. The durability and long-term effectiveness of this occlusion stent were confirmed in the 18-month follow-up ureterogram. A similar kind of occlusion stent with a wine-glass shape has been reported to successfully treat bronchopleural fistulas, following pneumonectomy [10]; in this study, a silicone-covered occlusion stent with a self-expandable feature and antimigra- 


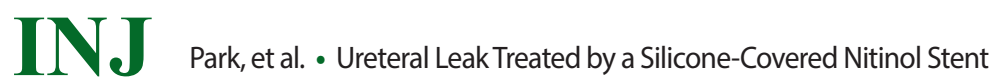

tion barbs was used for the remaining left main bronchus.

We propose the use of our specially designed, silicone-covered nitinol ureteral occlusion stent with a candy-wrapper configuration for the treatment of ureteral leakage. This stent is a less invasive, simple, and practical solution for patients with urinary leakage and in whom surgery is not indicated.

\section{REFERENCES}

1. Abboudi H, Ahmed K, Royle J, Khan MS, Dasgupta P, N’Dow J. Ureteric injury: a challenging condition to diagnose and manage. Nat Rev Urol 2013;10:108-15.

2. Koukouras D, Petsas T, Liatsikos E, Kallidonis P, Sdralis EK, Adonakis $\mathrm{G}$, et al. Percutaneous minimally invasive management of iatrogenic ureteral injuries. J Endourol 2010;24:1921-7.

3. Kim SK, Lee YR, Kyung MS, Choi JS. Transrenal ureteral occlusion with the use of microcoils in five patients with ureterovaginal fistulas. Abdom Imaging 2008;33:615-20.

4. Gunther R, Marberger M, Klose K. Transrenal ureteral emboliza- tion. Radiology 1979;132:317-9.

5. Moldwin RM, Smith AD. Percutaneous management of ureteral fistulas. Urol Clin North Am 1988;15:453-7.

6. Gunther RW, Klose KJ, Alken P, Bohl J. Transrenal ureteral occlusion using a detachable balloon. Urol Radiol 1984;6:210-4.

7. Papanicolaou N, Pfister RC, Yoder IC. Percutaneous occlusion of ureteral leaks and fistulae using nondetachable balloons. Urol Radiol 1985;7:28-31.

8. Farrell TA, Wallace M, Hicks ME. Long-term results of transrenal ureteral occlusion with use of Gianturco coils and gelatin sponge pledgets. J Vasc Interv Radiol 1997;8:449-52.

9. Pieper CC, Meyer C, Hauser S, Wilhelm KE, Schild HH. Transrenal ureteral occlusion using the Amplatzer vascular plug II: a new interventional treatment option for lower urinary tract fistulas. Cardiovasc Intervent Radiol 2014;37:451-7.

10. Chae EY, Shin JH, Song HY, Kim JH, Shim TS, Kim DK. Bronchopleural fistula treated with a silicone-covered bronchial occlusion stent. Ann Thorac Surg 2010;89:293-6. 